\title{
Recent advances in metamaterial klystrons
}

\author{
Xin Wang ${ }^{1}$, Xianfeng Tang ${ }^{2}$, Shifeng $\mathrm{Li}^{3}$, Junpu Ling ${ }^{4}$, Xuanming Zhang ${ }^{1}$, and Zhaoyun Duan ${ }^{1, *}$ \\ ${ }^{1}$ University of Electronic Science and Technology of China, Chengdu 610054, PR China \\ 2 School of Physical Science and Technology, Southwest Jiaotong University, Chengdu 610031, PR China \\ ${ }^{3}$ Institute of Applied Electronics, China Academy of Engineering Physics, Mianyang 621900, PR China \\ ${ }^{4}$ College of Advanced Interdisciplinary Studies, National University of Defence Technology, Changsha 410073, PR China
}

Received: 10 November 2020 / Accepted: 1 January 2021

\begin{abstract}
As a kind of artificially structured media, electromagnetic metamaterials (MTMs) have exotic electromagnetic properties that are not found or difficult to achieve in natural materials. This class of metal/ dielectric-structured artificial media has attracted great attention during the past two decades and made important breakthroughs. A variety of passive and active devices based on MTMs have been developed rapidly. Especially MTM klystrons, which show very remarkable advantages, including miniaturization, high gain, and high efficiency in the microwave band. MTM extended interaction klystrons creatively combine the advantages of MTMs, extended interaction technology, and klystrons. It provides a new design idea for the development of brand-new klystrons with high performance. In this review paper, we report the recent advances in MTM klystrons including MTM extended interaction oscillator and MTM extended interaction klystron amplifier. Furthermore, the prospects and challenges of MTM klystrons are discussed. Finally, the development trend is concluded.
\end{abstract}

Keywords: Metamaterial / klystron / miniaturization / high power / high efficiency

\section{Introduction}

Metamaterials (MTMs) were proposed theoretically by Veselago in left-handed material defined with simultaneously negative permittivity $(\varepsilon)$ and negative permeability $(\mu)$ in 1967, where the electric field vector $\boldsymbol{E}$, the magnetic field vector $\boldsymbol{H}$, and the wave vector $\boldsymbol{k}$ satisfy the left-handed law in this medium [1]. Until the year of 2000, MTMs were first realized in the microwave frequency band based on the effective medium theory $[2,3]$. Since then, various MTMs and their exotic electromagnetic (EM) properties such as negative refractive index, reversed Doppler effect, and reversed Cherenkov radiation have been widely investigated [4-8]. Meanwhile, the engineering concepts and applications of MTMs have gradually become widespread, also, the unit cell of MTMs has been changed from the split-ring resonator (SRR), metal wires to their complementary structure (CSRR/CeSRR), and various deformation structures [9-15]. At the same time, the original MTMs have gradually evolved into broader synthetic materials, such as low-loss MTMs, all-metal MTMs, broad band MTMs, metasurface, coding MTMs, and information MTMs [15-22]. In the

\footnotetext{
* e-mail: zhyduan@uestc. edu.cn
}

development, MTMs were broadly defined as a kind of artificially structured media composed with periodically or non-periodically arranged subwavelength unit cells, which can achieve exotic EM properties beyond the limits of naturally occurring materials [23,24]. Different from natural materials, the EM properties of MTMs can be controlled by changing the shape, sizes, and arrangement of MTM unit cells. Especially, due to the elaborated structure design, MTMs can provide a great freedom to manipulate EM waves. As a consequence, a variety of passive and active metadevices such as MTM-inspired antennas, filters, couplers, absorbers [25-31], and MTMs-based vacuum electron devices (VEDs), including traveling-wave tubes (TWTs) [32-34], resistive wall amplifiers [35], backwardwave oscillators (BWOs) [36-46], klystrons [47,48], and accelerators $[49,50]$ have attracted considerable attention.

Klystrons are widely used in the military and civilian fields, such as high power radars, particle accelerators, radio-astronomy, industrial heating, medical imaging, etc., owing to their high power, high efficiency, high gain, and low phase noise [51-55]. With the development of science and technology, the higher performance klystrons are required to meet the application requirements in basic scientific facilities [56-58]. However, the conventional klystrons have large size and weight in low frequency 
band. Thus, based on the characteristics of MTMs with miniaturization, narrow band operation, and strong resonance, the creative combination of MTMs and klystrons has provided a new design idea for the development of brand-new MTM extended interaction klystrons (MEIKs) $[47,48]$.

As MTMs comprise the interdisciplinary and rapidly growing research fields, different review articles focused on different areas [23-26,57,58]. Here, we provide our prospects on the field of emerging MTM klystrons by reviewing the research progress in recent years. In Section 2, the MTMs suitable for VEDs are presented, and the EM characteristics of cavity loaded with MTMs are introduced. In Section 3, we review the MTMs extended interaction oscillators (MEIOs) and their performance. And then, we present the development status of MEIKs and their potential applications in Section 4. We also present the prospects and challenges of MTM klystrons. At last, we give concluding remarks and an outlook in the research directions of MTM klystrons.

\section{MTM extended interaction resonant cavity}

The loss of original MTMs comes from the metal resonators and the dielectric substrate, which limits the applications of MTM since the most dielectric substrates are not suitable for vacuum environments. Thus, all-metal MTMs become necessary for the development of klystrons due to their low-loss and vacuum demand. The unit cell of MTMs is composed of electric dipoles and magnetic dipoles. Also, these dipoles can be provided through SRRs, metal wires, CSRRs/CeSRRs, or slot lines. Besides, an empty waveguide operating below the cutoff frequency can be treated as electric or magnetic dipoles under the different operation modes, namely, the effective permeability is negative for the TM mode, and the effective permittivity is negative for the TE mode $[59,60]$. Therefore, the different all-metal MTM elements are listed in Figure 1. The novel EM properties can be realized by combining the specific MTM elements.

In 2016, Wang et al. proposed an all-metal waveguidebased MTMs and investigated the dispersion and transmission properties by both simulation and experiment [15]. And then a pencil beam BWO with miniaturization and high-efficiency was proposed and verified by the experiments in 2020 [40,41]. Furthermore, Wang et al. studied the dispersion, interaction impedance, mode distribution, and propagation properties of the MTM slow-wave structure with equivalent circuit model [61]. Also, the effects of MTMs on the mode distribution were studied. The different field distributions of the slot mode and cavity mode are shown in Figure 2, which indicate that MTM slow-wave structure can provide a strong longitudinal electric field for the energy conversion between the electron beam and the EM wave, and is suitable for constructing extended interaction resonant cavity (EIRC) [61,62].

The performance of klystron such as gain and electronic efficiency largely depends on the interaction structure of multiple resonate cavities. The electron beam is centralized

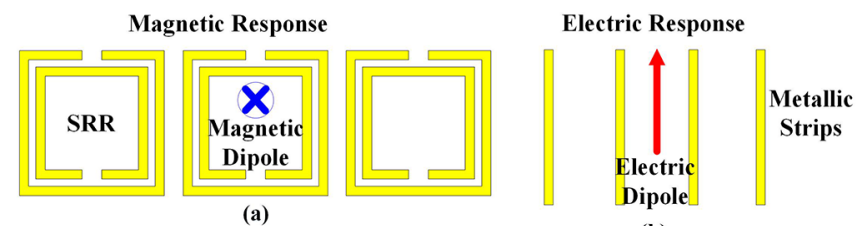

(a)

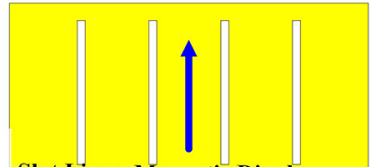

Slot Lines Magnetic Dipole
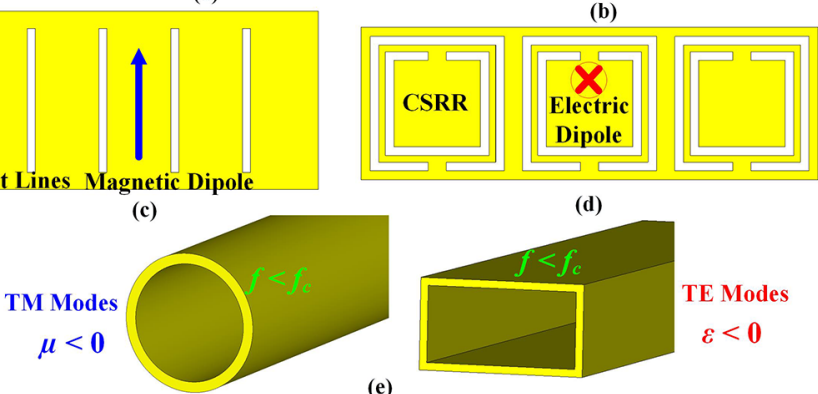

(d)

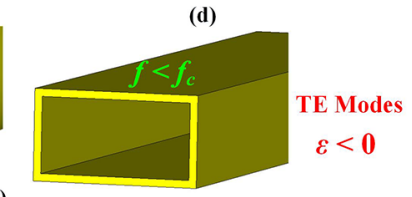

Fig. 1. MTM elements. (a) SRRs, it can be equivalent to a magnetic dipole, (b) metal wires, it can be equivalent to an electric dipole, (c) slot lines, it can be equivalent to a magnetic dipole, (d) CSRRs, it can be equivalent to an electric dipole, and (e) the empty waveguide operating below the cutoff frequency, the effective permeability is negative for the TM mode, the effective permittivity is negative for the TE mode.

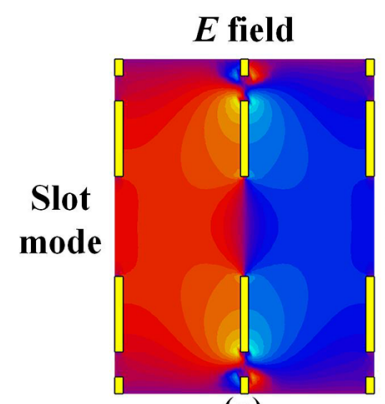

(a)

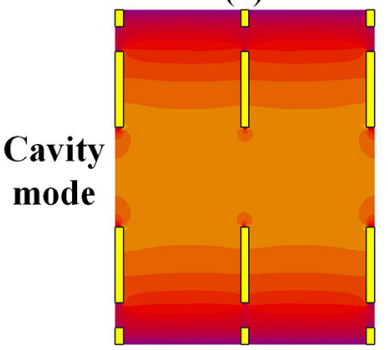

(c)

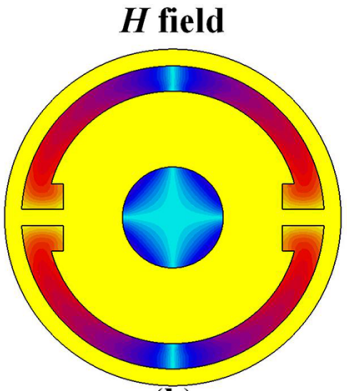

(b)

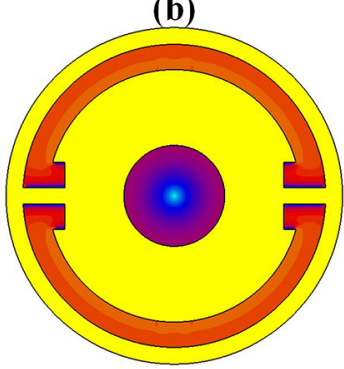

(d)

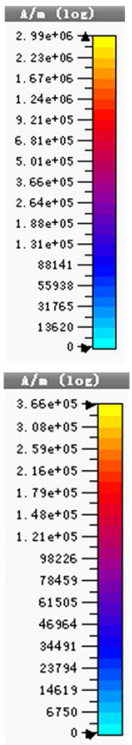

0
Fig. 2. Contours of the longitudinal electric field and transverse magnetic field. (a) Longitudinal electric field of the slot mode and (b) transverse magnetic field of the slot mode, (c) longitudinal electric field of the cavity mode, and (d) transverse magnetic field of the cavity mode [61].

modulation by high frequency field in the cavity. Also, the EIRC combines the advantages of slow-wave structure and cavity, which has larger effective characteristic impedance $\left(M^{2} R / Q\right)$ than the single gap resonant cavity [63]. Wang et al. proposed an MTM EIRC in the first time, as shown in Figure 3, which consists of a circular waveguide loaded with MTM arrays $[47,48]$. To explore the structural sizes and 


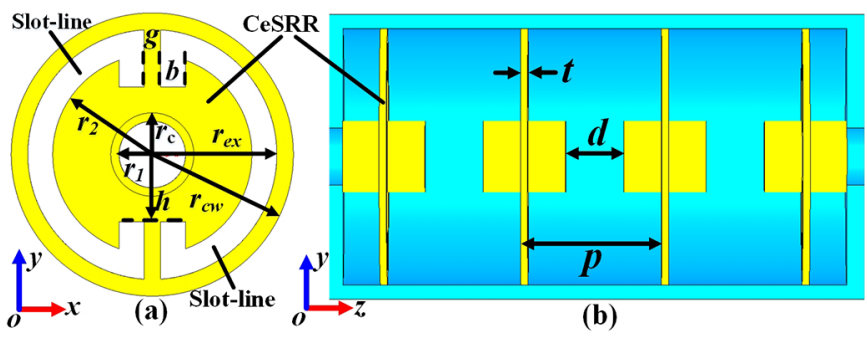

Fig. 3. (a) A CeSRR unit cell and (b) 3-gap MTM EIRC, where $r_{1}=6.5 \mathrm{~mm}, r_{2}=12 \mathrm{~mm}, r_{\mathrm{ex}}=16.5 \mathrm{~mm}, r_{\mathrm{cw}}=18.5 \mathrm{~mm}$,

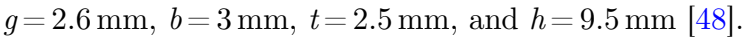

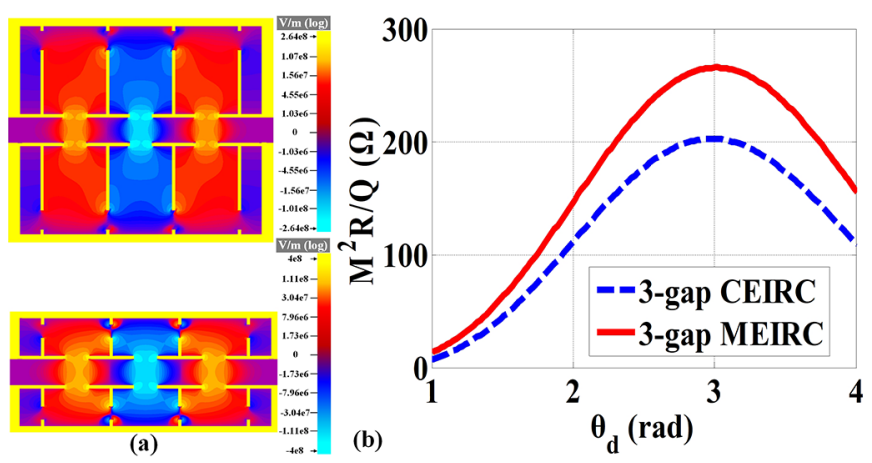

Fig. 4. Simulated and calculated results. (a) Fundamental mode longitudinal electric field contours of the 3-gap conventional EIRC (upper) and the 3-gap MTM EIRC (lower), and (b) $M^{2} R / Q$ versus gap transit angle [48].

$M^{2} R / Q$ between the conventional EIRC and MTM EIRC, the EM characteristics of the 3-gap conventional EIRC and 3-gap MTM EIRC are compared by using eigenmode simulation and calculation.

The eigenmode simulation and calculation results of the 3-gap conventional EIRC and 3-gap MTM EIRC are shown in Figure 4 [48]. Their fundamental modes are $\pi$ mode, and the maximum $M^{2} R / Q$ of MTM EIRC and conventional EIRC are $265 \Omega$ and $202 \Omega$, respectively. The corresponding cavity inner diameters are $33 \mathrm{~mm}(\sim \lambda / 4$, where $\lambda$ is the wavelength in free space) and $65 \mathrm{~mm}(\sim \lambda / 2)$, respectively. That is, the cavity inner diameter of the MTM EIRC is $\sim 1 / 2$ of its counterpart of the conventional EIRC, and the MTM EIRC has higher $M^{2} R / Q$ over the conventional EIRC [48]. The higher $M^{2} R / Q$ means the MTM EIRC has stronger velocity modulation to the electron beam, which can provide a larger fundamental wave modulation current in a shorter drift-tube, thereby shortening the whole length of the MEIK and achieving longitudinal miniaturization.

\section{MTM extended interaction oscillators}

Slow-wave structure with short circuitsat both ends can construct a multi-gap EIRC [63]. Compared with a singlegap resonant cavity, EIRC has higher $M^{2} R / Q$. At the same time, as the number of periods increases, the frequency gap of the adjacent modes becomes smaller, which can easily

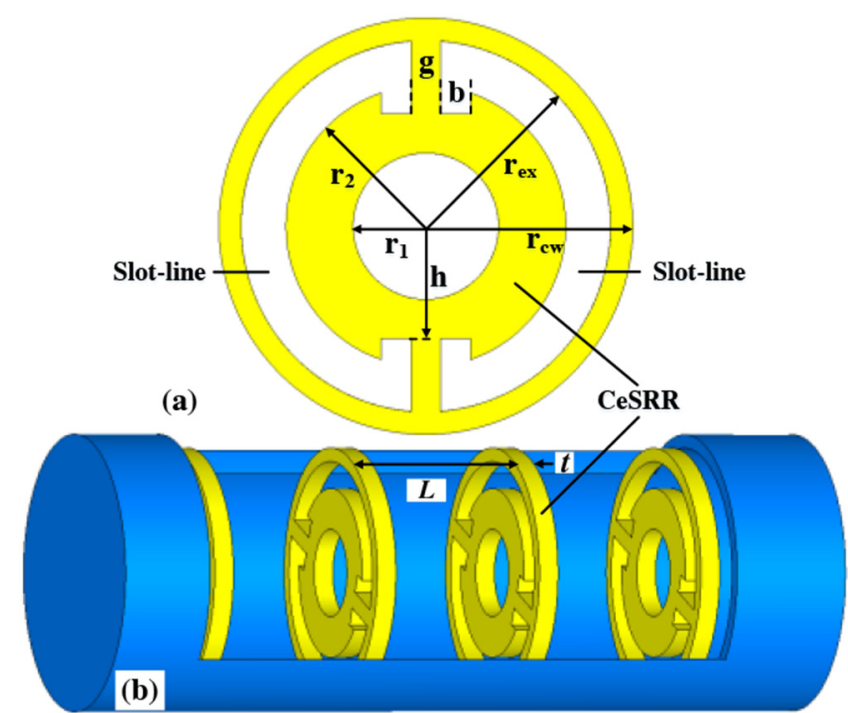

Fig. 5. (a) CeSRR unit cell and (b) 3-gap MTM EIRC, where $r_{1}=6.5 \mathrm{~mm}, r_{2}=12 \mathrm{~mm}, r_{\mathrm{ex}}=16.5 \mathrm{~mm}, r_{\mathrm{cw}}=18.5 \mathrm{~mm}$, $g=2.6 \mathrm{~mm}, b=3 \mathrm{~mm}, t=2.5 \mathrm{~mm}$, and $h=9.5 \mathrm{~mm} \mathrm{[64]}$.

cause oscillation of klystron amplifier. On the contrary, the oscillators are easily achieved according to these features, especially in MTM EIRC which has shorter circuit length than conventional BWOs. MTM EIRC shows the lateral miniaturization, narrow operation bandwidth, and strong local electric/magnetic fields. It can be predicted that MEIO can simultaneously have the characteristics of miniaturization and high electronic efficiency because MEIO can combine the advantages of MTMs and extended interaction technology.

In 2020, Wang et al. investigated the EM characteristics of multiple-gap MEIO by using the theory and simulation analysis [64]. As shown in Figure 5, the CeSRR unit cells filled in a circular waveguide with short circuits at both ends construct a 3-gap MTM EIRC. Here, the power exchange function $F_{N}(\theta)$ of an N-gap MTM EIRC is adopted to characterize the degree of beam-wave interaction of MTM EIRC with different gap numbers [64].

The calculated results show that as the number of periods increases, the magnitude of $F_{N}(\theta)$ increases, as shown in Figure 6. It means that the electron beam has a more sufficient energy exchange with high frequency field in the MTM EIRC. The plots of $F_{N}(\theta)$ reveal a possibility that the energy exchange of the beam-wave interaction can be largely realized in a very short distance, that is, the MTM EIRC is conducive to the development of klystron oscillators with miniaturization and high electronic efficiency.

Furthermore, an S-band MW-level MEIO is analyzed, and the beam-wave interaction simulation is carried out by using CST Particle Studio. As shown in Figure 7a, a waveguide coupler is used to connect the 5 -gap MEIO with the standard rectangular waveguide BJ32, which can ensure the power capacity with MW-level at the output port $[64,65]$. Under the condition that the beam voltage and current are taken as $130 \mathrm{kV}, 80 \mathrm{~A}$, respectively, and the 


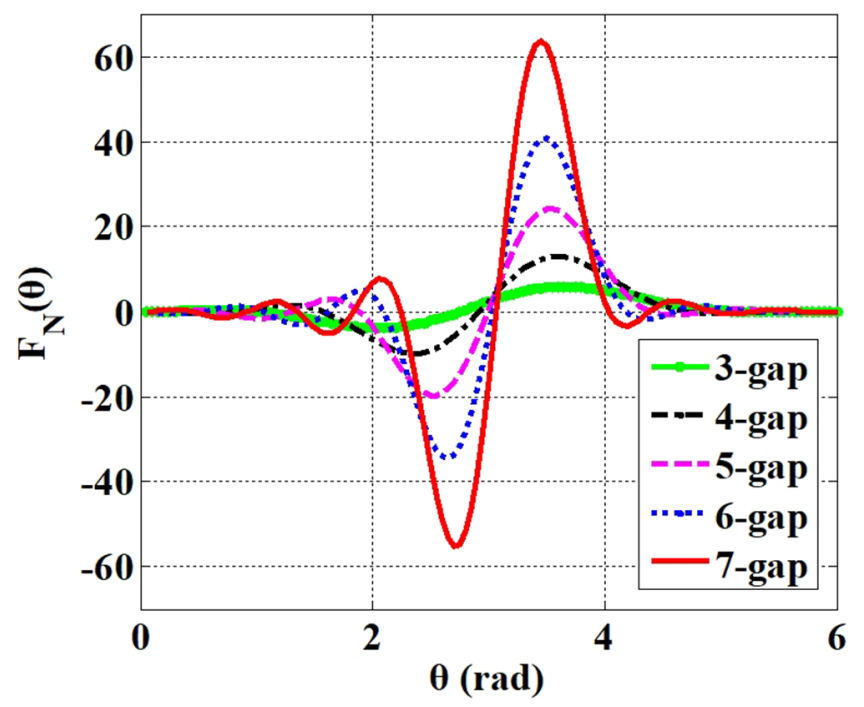

Fig. 6. Power exchange function $F_{\mathrm{N}}(\theta)$ versus gap transit angle $\theta(\theta=\beta L, \beta$ is the phase constant and $L$ is the period $)$, also, $F_{\mathrm{N}}(\theta)$ $<0$ and $F_{\mathrm{N}}(\theta)>0$ stand for the status of oscillation and amplification, respectively [64].

focusing magnetic field is $2000 \mathrm{G}$, the simulated results predict that a 5-gap MEIO with a waveguide coupler can achieve about peak output power of $10 \mathrm{MW}$ (average power of $5 \mathrm{MW}$ ) and electronic efficiency up to $48 \%$ at $2.866 \mathrm{GHz}$. These results indicate that, as a microwave source, the MEIO has shorter longitudinal length than the conventional BWOs because of the resonant cavity structure. Besides, the introduction of MTMs has realized the horizontal miniaturization of the MEIO. In summary, the combination of MTMs and extended interaction techlonogy has opened a new door for the new design of MEIO with miniaturization and high electronic efficiency.

\section{MTM extended interaction klystrons}

The previous research results show that the MEIO exhibits excellent performance in terms of structural size and electronic efficiency. Similarly, MEIK using MTM EIRC as the interaction structure, the amplification mechanism depends on the properties of the independent MTM EIRCs. Also, the miniaturization of the interaction structure is more significant in the lower microwave bands. Next, the recent advances in the MEIKs are presented.

Since 2016, Duan's group has explored the possibility of combining MTMs and klystron amplifier [66]. They proposed a multiple-gap MTM EIRC with MTM unit cells, then developed a 3-cavity MEIK with ring couplers and achieved the initial miniaturization and high gain [47]. Furthermore, Wang et al. investigated the 2-gap and 3-gap MTM EIRCs with rod coupler by simulation and experiment. The simulation model and the sample of the MTM EIRCs are shown in Figure 8 [48]. The measured resonant frequencies and external quality factors of the MTM EIRCs are in agreement with full-wave simulations.

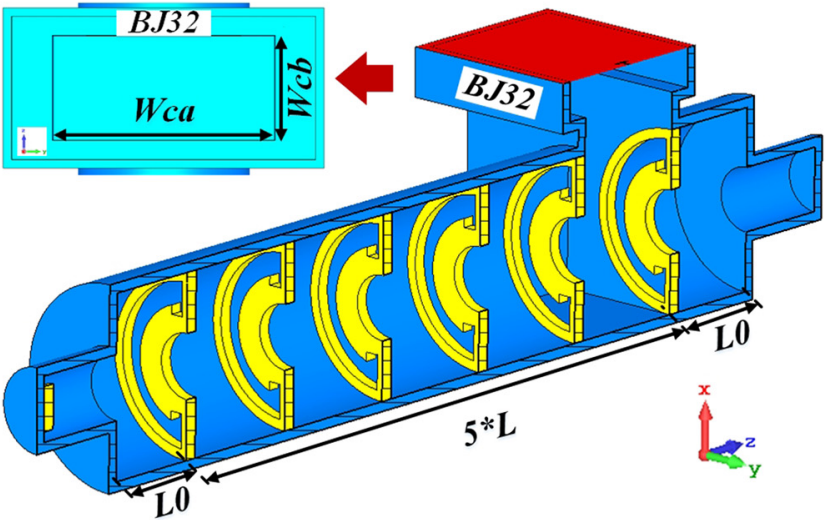

(a)

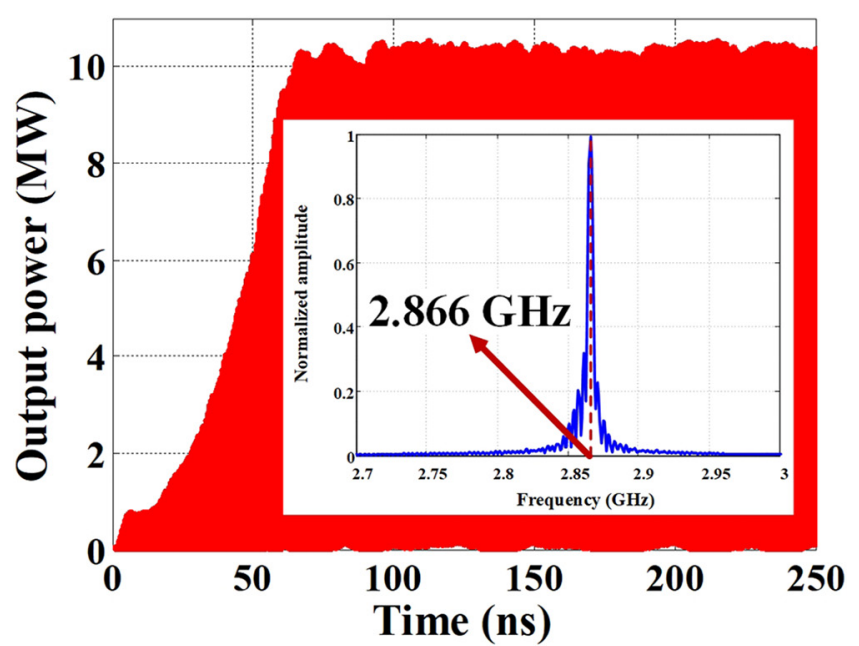

(b)

Fig. 7. Simulation model and the PIC beam-wave interaction results in CST. (a) Schematic of the 5-gap MEIO, where the inner diameter is $37 \mathrm{~mm}(\sim \lambda / 2.83)$ and the longitudinal interaction length is about $180 \mathrm{~mm}(\sim 1.2 \lambda)$, (b) peak output power, and spectrum (inset) of the output signal [64].

Thus, the simulation and test results fully indicate the feasibility of miniaturization in the MEIK by utilizing the MTM EIRC as the interaction structure.

Based on the aforementioned EM characteristics of MTM EIRC, the beam-wave interaction of the 3-cavity MEIK is carried out by using the particle-in-cell (PIC) simulation to predict the performance of the MEIK. The schematic of the 3 -cavity MEIK with input rod coupler and waveguide output coupler is shown in Figure 9 [48]. The attenuator, located in the left side of the buncher, is adopted to reduce the Q-value and suppress the intrinsic oscillation from high-order modes.

The EM wave amplification of the 3-cavity MEIK is predicted by PIC simulation, and the results are shown in Figure 10a. The beam voltage is $30 \mathrm{kV}$ and the current is $3 \mathrm{~A}$. The results show that the saturated output power is $56 \mathrm{~kW}$ at $2.453 \mathrm{GHz}$, the corresponding gain is $47 \mathrm{~dB}$, and 


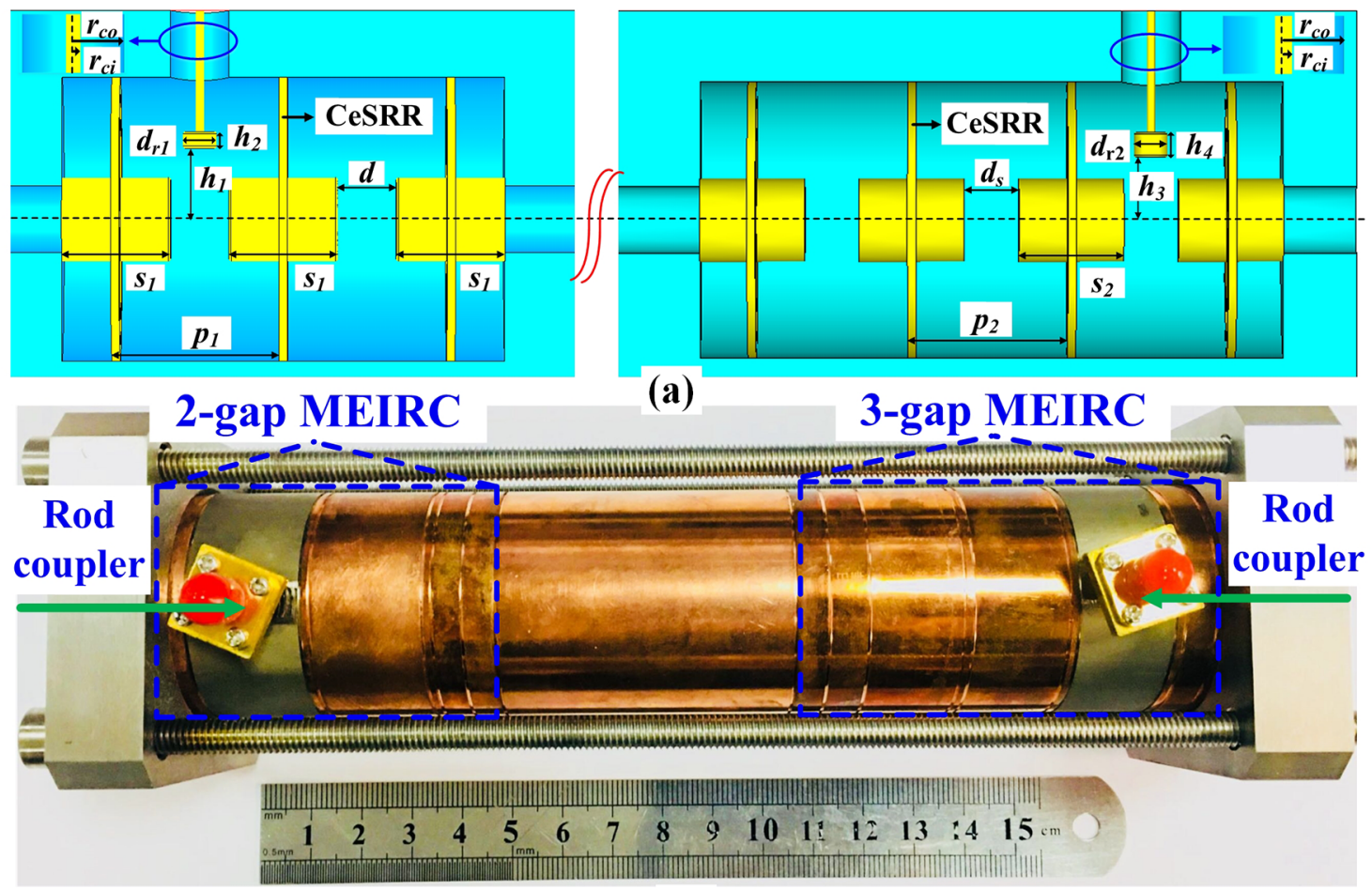

(b)

Fig. 8. Simulation model and sample of the MTM EIRCs. (a) 2-gap MTM EIRC (left) and 3-gap MTM EIRC (right) with rod couplers and (b) photo of the assembled MTM EIRCs. The parameters are set as $p_{1}=20 \mathrm{~mm}, s_{1}=13 \mathrm{~mm}, d=7 \mathrm{~mm}, h_{1}=11.5 \mathrm{~mm}, h_{2}=2 \mathrm{~mm}$, $d_{\mathrm{r} 1}=4 \mathrm{~mm}, r_{\mathrm{co}}=2.7 \mathrm{~mm}, r_{\mathrm{ci}}=0.65 \mathrm{~mm}, p_{2}=19 \mathrm{~mm}, s_{2}=12.5 \mathrm{~mm}, d_{\mathrm{s}}=6.5 \mathrm{~mm}, h_{3}=11 \mathrm{~mm}, h_{4}=3 \mathrm{~mm}$, and $d_{\mathrm{r} 2}=4 \mathrm{~mm}[48]$.

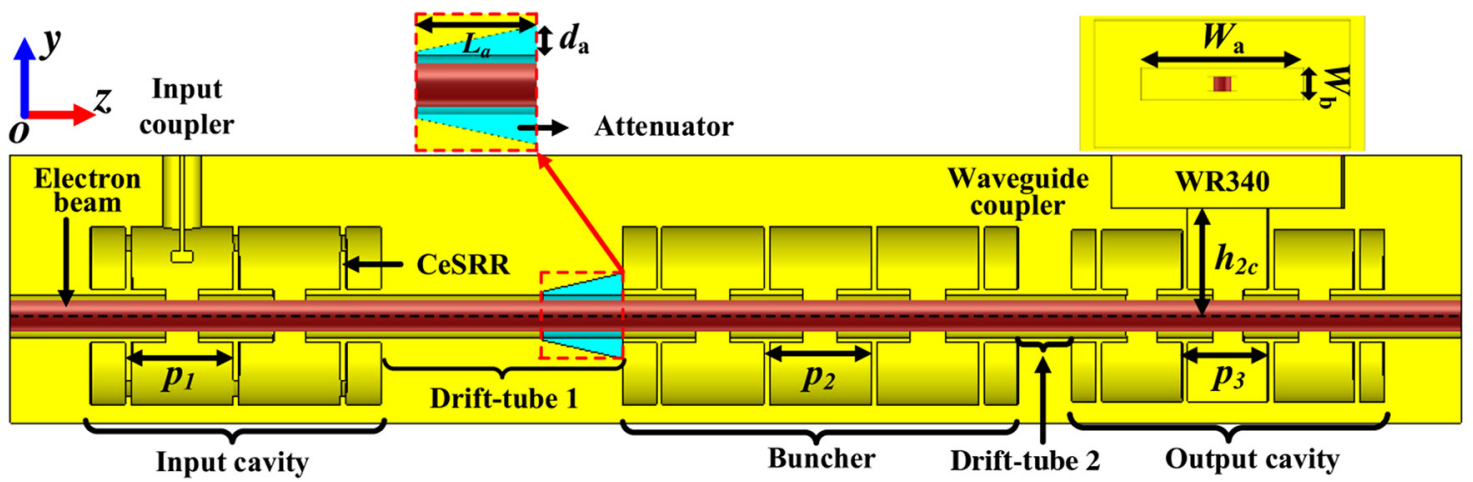

Fig. 9. Schematic of the 3-cavity MEIK in the yoz plane. The periods of the input cavity, mid-cavity (buncher), and output cavity are $p_{1}=20 \mathrm{~mm}, p_{2}=20 \mathrm{~mm}$, and $p_{3}=16 \mathrm{~mm}$, respectively, and the parameters of the waveguide coupler are $W_{\mathrm{a}}=50.5 \mathrm{~mm}, W_{\mathrm{b}}=16 \mathrm{~mm}$, and $h_{2 \mathrm{c}}=19 \mathrm{~mm}$. The length of drift-tube 1 and drift-tube 2 are 55 and $20 \mathrm{~mm}$, respectively, and the entire interaction length is $\sim 210 \mathrm{~mm}(\sim 1.72 \lambda)[48]$.

the electronic efficiency is $62 \%$ [48]. Furthermore, the electronic efficiency and gain are obtained by changing input power as shown in Figure 10b [48]. If the multistage depressed collector is used in the future, the total efficiency can be further improved. Therefore, the 3-cavity MEIK exhibits its advantages in miniaturization, power, efficiency, and gain. In summary, the state of the art of the MTM klystrons is listed in Table 1.

\section{Prospects and challenges}

Under the impetus of scientific and technological progress and practical applications, klystrons are developing towards high power, high efficiency, high gain, miniaturization, high reliability, and long lifetime [51-53]. In recent years, the development of MTM klystrons has provided a new design idea for exploring higher performance. MTM 


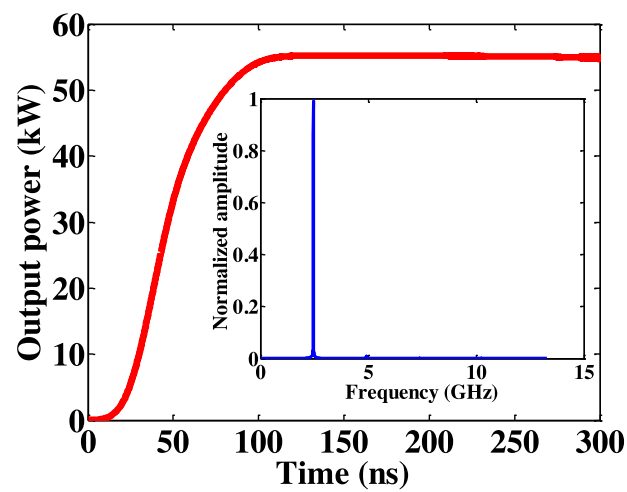

(a)

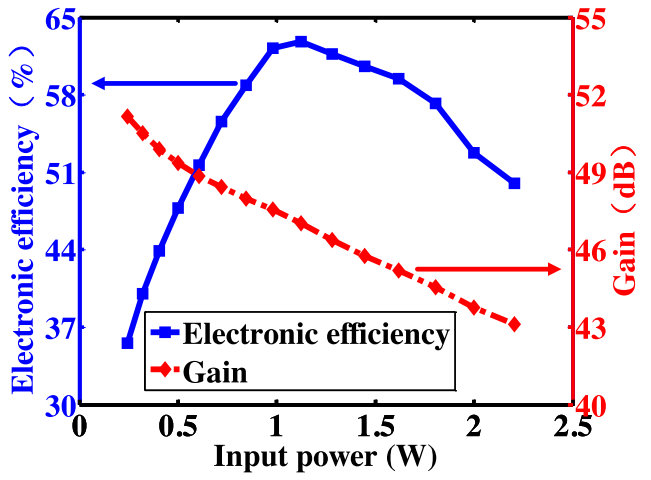

(b)

Fig. 10. PIC simulation results, (a) saturated output power of the 3-cavity MEIK at 2.453 GHz (inset), and (b) electronic efficiency and gain versus input power [48].

Table 1. Features of typical MTM klystrons.

\begin{tabular}{|c|c|c|c|}
\hline & Category & Function & Feature \\
\hline & MEIO & $\begin{array}{l}\text { (i) Improving characteristic impedance } \\
\text { (ii) Shortening the interaction length } \\
\text { (iii) Reducing the transverse dimensions of the cavity }\end{array}$ & $\begin{array}{l}\text { (i) Miniaturization } \\
\text { (ii) High power } \\
\text { (iii) High efficiency } \\
\text { (iv) Narrow tuning bandwidth }\end{array}$ \\
\hline MTM Klystrons & MEIK & $\begin{array}{l}\text { (i) Reducing the transverse dimensions of the cavity } \\
\text { (ii) Improving characteristic impedance }\end{array}$ & $\begin{array}{l}\text { (i) Miniaturization } \\
\text { (ii) High power } \\
\text { (iii) High gain } \\
\text { (iv) High efficiency } \\
\text { (v) Narrow operation bandwidth }\end{array}$ \\
\hline
\end{tabular}

klystrons show the following advantages: (1) The transverse size of the MTM EIRC is only $2 / 3$ to $1 / 2$ or even smaller than that of the traditional reentry EIRC. Thus, MEIK shows the significant feature inminiaturization. (2) MTM EIRC has the higher $M^{2} R / Q$ than the conventional EIRC. This fact means that the MTM EIRC has stronger velocity modulation to the electron beam and potential higher electric efficiency. (3) Compared with the conventional oscillators, MEIOcan achieve miniaturization and high electronic efficiency.

In addition to the advantages mentioned above, MTMinspired klystrons are faced with these challenges: (1) MEIK highlights the characteristics of horizontal miniaturization, however, we find that the longitudinal length does not decrease significantly. (2) The introduction of MTMs maybe increase the risk of higher voltage breakdown under the condition of higher power. (3) MTM klystrons have the feature of narrow band, which limits their applications.

Based on the advances in MTM klystrons, it is expected that MTM klystrons will develop towards miniaturization, high power, high gain, and high efficiency for future accelerators, controlled thermonuclear fusion devices, industrial heating, medical imaging and so on.

\section{Conclusion}

Klystrons continue to play an important role in high-power microwave sources and amplifier applications. As mentioned before, the future of MTM klystrons will depend on the advances in other technologies. MTMs suitable for vacuum will be tailored to meet the requirements of klystron performance. It will explore higher performances based on the application demands of basic scientific facilities and medical imaging.

At the same time, with the devolpment of theory, simulation technologies, experimental research, and the practical application demands, the trends of MTM klystrons in the future can be forecasted: (1) Explore new EM MTMs suitable for VEDs and high power to solve the potential problems such as high voltage breakdown and heat dissipation caused by high power. (2) Make the best of the miniaturization characteristics of MTMs to develop high peak power klystrons in $\mathrm{P}$-band to X-band from several MW to GW-level, and high average power MTM klystrons (or MEIKs) in hundreds kW-level (several MW) for particle accelerators. (3) Carry out the experimental research on miniaturized high electronic efficiency MEIO and explore the miniaturized MEIO with GW-level peak 
power. (4) Develop the theoretical and experimental research on millimeter wave and terahertz wave MTM klystrons.

This work was supported in part by the National Natural Science Foundation of China under Grant Nos. 61871095, 61921002, and 61531010, and the CNS project under Grant No. Y03111023901014005. The corresponding author Zhaoyun Duan would like to thank Prof. Runhua Fan at the Shanghai Maritime University and Prof. Yang Hao at the Queen Mary University of London for their invitation.

\section{References}

1. V.G. Veselago, Sov. Phys. Uspekhi. 47, 509 (1968)

2. D.R. Smith, N. Kroll, Phys. Rev. Lett. 85, 2933 (2000)

3. D.R. Smith, W.J. Padilla, D.C. Vier, S.C. Nemat-Nasser, S. Schultz, Phys. Rev. Lett. 84, 4184 (2000)

4. Z. Duan, B.I. Wu, J. Lu, J.A. Kong, M. Chen, J. Appl. Phys. 104, $063303(2008)$

5. Z. Duan, C. Guo, M. Chen, Opt. Express 19, 13825 (2011)

6. R.A. Shelby, D.R. Smith, S. Schultz, Science 292, 77 (2001)

7. N. Seddon, T. Bearpark, Science 302, 1537 (2003)

8. Z. Duan et al., Nat. Commun. 8, 14901 (2017)

9. J.B. Pendry, A. Holden, D. Robbins, W. Stewart, J. Phys.: Condens. Matter 10, 4785 (1998)

10. J.B. Pendry, A.J. Holden, D.J. Robbins, W.J. Stewart, IEEE Trans. Microw. Theory Tech. 47, 2075 (1999)

11. D.R. Smith et al., Phys. Rev. Lett. 84, 4184 (2000)

12. L. Liang et al., Sci. China Inf. Sci. 56, 120412 (2013)

13. X. Lu, M.A. Shapiro, R.J. Temkin, Phys. Rev. Spec. Top. Accel. Beams. 18, 1 (2015)

14. Y. Hao, R. Mittra, FDTD Modelling of Metamaterials: Theory and Applications ( Artech House, Norwood, 2008)

15. Y. Wang et al., Appl. Phys. Lett. 107, 1 (2015)

16. Z. Duan, J.S. Hummelt, M.A. Shapiro, R.J. Temkin, Phys. Plasmas 21, 103301 (2014)

17. R. Liu, Q. Cheng, J.Y. Chin, J.J. Mock, T. Cui, D.R. Smith, Opt. Express 17, 21030 (2009)

18. S. Walia et al., Appl. Phys. Rev. 2, 011303 (2015)

19. X. Fu, T. Cui, Prog. Quantum Electron. 67, 100223 (2019)

20. C.D. Giovampaola, N. Engheta, Nat. Mater. 13, 1115 (2014)

21. C.L. Holloway, E.F. Kuester, J.A. Gordon, J.O. Hara, J. Booth, D.R. Smith, IEEE Antennas Propag. Mag. 54, 10 (2012)

22. T.J. Cui, M. Qi, X. Wan, J. Zhao, Q. Cheng, Light Sci. Appl. 3, 218 (2014)

23. R.S. Kshetrimayum, IEEE Potentials 23, 44 (2005)

24. Y. Dong, T. Itoh, IEEE Microw. Mag. 13, 2 (2012)

25. N.I. Zheludev, Y.S. Kivshar, Nat. Mater. 11, 917 (2012)

26. H. Chen, IEEE Photonics Soc. Winter Top. Meet. Ser. WTM 444, 28 (2010)

27. T. Cui, D.R. Smith, R. Liu, Metamaterials: theory, design, and applications (Springer, New York, Dordrecht, Heidelberg, London, 2009)
28. Q. Zhang, H. Zhang, J. Yin, B. Pan, T. Cui, Sci. Rep. 6, 28256 (2016)

29. H. Zhang, S. Liu, X. Shen, L. Chen, L. Li, T. Cui, Laser Photonics Rev. 9, 83 (2015)

30. F. Wang et al., Prog. Electromagn. Res. C 84, 61 (2018)

31. F. Wang et al., Int. J. Antennas Propag. 2019, 1 (2019)

32. Y.S. Tan, R. Seviour, Europhys. Lett. 87, 34005 (2009)

33. L. Chao, S. Guo, M.N. Afsar, J.R. Sirigiri, Metamaterial based negative refractive index traveling wave tube, 2013 19th IEEE Pulsed Power Conference (PPC), San Francisco, CA, 2013, pp. 1-5, doi: 10.1109/PPC.2013.6627598

34. D. Starinshak, J. Wilson, NASA/TP. (Report) (2007) 214701

35. T. Rowe, J.H. Booske, N. Behdad, IEEE Trans. Plasma Sci. 43, $2123(2015)$

36. D. Shiffler, J. Luginsland, D.M. French, J. Watrous, IEEE Trans. Plasma Sci. 38, 1462 (2010)

37. J.K. So et al., Appl. Phys. Lett. 97, 97 (2010)

38. S.C. Yurt, M.I. Fuks, S. Prasad, E. Schamiloglu, Phys. Plasmas 23, 123115 (2016)

39. J.S. Hummelt, X. Lu, H. Xu, I. Mastovsky, M.A. Shapiro, R.J. Temkin, Phys. Rev. Lett. 117, 1 (2016)

40. Y. Wang et al., IEEE Trans. Electron Devices 63, 3747 (2016)

41. A.B. De Alleluia et al., IEEE Trans. Plasma Sci. 48, 1 (2020)

42. X. Tang et al., IEEE Trans. Electron Devices 64, 2376 (2017)

43. J. He, J. Ling, B. Deng, O. Dai, W. Xu, L. Wang, Phys. Plasmas 26, 23104 (2019)

44. H. Seidfaraji, A. Elfrgani, C. Christodoulou, E. Schamiloglu, Phys. Plasmas 26, 073105 (2019)

45. G. Wu et al., IEEE Trans. Electron Devices 65, 1172 (2018)

46. P. Narasimhan, S. Jain, N. Gurjar, N. Kumar, S.K. Ghosh, IEEE Trans. Electron Devices 67, 1227 (2020)

47. X. Wang, Z. Duan, F. Wang et al., A miniaturized high-gain, high-efficiency metamaterial assited S-band extended interaction klystron, 2019 International Vacuum Electronics Conference (IVEC), Busan, Korea (South), 2019, pp.1-2, doi: 10.1109/IVEC.2019.8744761

48. X. Wang et al., IEEE Electron Device Lett. 41, 1580 (2020)

49. X. Lu et al., Appl. Phys. Lett. 116, 264102 (2020)

50. X. Lu et al., Appl. Phys. Lett. 117, 073502 (2020)

51. R.K. Parker, R.H. Abrams, B.G. Danly, B. Levush, IEEE Trans. Microw. Theory Tech. 50, 835 (2002)

52. J. Qiu et al., IEEE Microw. Mag. 10, 38 (2009)

53. B.N. Basu, S.K. Datta, J. Electromagnet. Wave Appl. 5071, 1771 (2017)

54. S. Li et al., IEEE Trans. Electron Devices 66, 2758 (2019)

55. S. Li et al., Phys. Plasmas 26, 43107 (2019)

56. G. Caryotakis, National Accelerator Lab. Menlo Park, Ca (United States) (2004)

57. Z. Duan et al., IEEE Trans. Electron Devices 66, 1 (2017)

58. R. Guha, X. Wang, A.K. Varshney, Z. Duan, M.A. Shapiro, B.N. Basu, Metamaterials: technology and applications (CRC Press, Boca Raton, 2020) (to be published) 
59. R. Marqués, J. Martel, F. Mesa, F. Medina, Phys. Rev. Lett. 89, 5 (2002)

60. J. Esteban et al., IEEE Trans. Microw. Theory Tech. 53, $1506(2005)$

61. X. Wang et al., IEEE Trans. Microw. Theory Tech. 67, 2238 (2019)

62. X. Wang, Z. Duan, F. Wang et al., High frequency characteristics of a metamaterial slow wave structure, 2018 IEEE International Vacuum Electronics Conference (IVEC), Monterey, CA, 2018, pp. 345-346, doi: 10.1109/ IVEC.2018.8391518
63. M. Chodorow, T. Wessel-berg, IRE Trans. Electron Devices 8, $44(1961)$

64. X. Wang, H. Luo, X. Zhang et al., Design of a compact and high-efficiency metamaterial extended interaction oscillator, 2020 IEEE International Vacuum Electronics Conference (IVEC), Virtual, 2020 (to be published)

65. Z. Duan et al., in Proceedings of URSI Regional Conference on Radio Science 2020, Varanasi, 2020, p. 1

66. Z. Duan et al., in Proceedings of the International Vacuum Electronics Conference, London, 2017, p. 1

Cite this article as: Xin Wang, Xianfeng Tang, Shifeng Li, Junpu Ling, Xuanming Zhang, Zhaoyun Duan, Recent advances in metamaterial klystrons, EPJ Appl. Metamat. 8, 9 (2021) 